\title{
OBSERVACIONES SOBRE ALLIUM ROUYI GAUTIER
}

\author{
Baltasar CABEZUDO, Julio PASTOR, M. Mar TRIGO y \\ José María NIETO CALDERA
}

\begin{abstract}
RESUMEN. Se ha localizado una población de Allium rouyi Gautier, especie considerada como extinta. En base al material recolectado se hace una nueva descripción y un estudio de las semillas y polen.
\end{abstract}

Palabras clave. Allium rouyi, Lilicaceae, polen, semilla, Andalucía.

ABSTRACT. A population of Allium rouy Gautier, considered as an extinguished specie, has been recently found. So a new description and study of the seeds and the pollen grains of this taxon has been included.

Key words. Allium rouyi, Lilicaceae, pollen, seed, Andalucía.

\section{INTRODUCCIÓN}

La realización de estudios sobre la biología de especies endémicas y en peligro de extinción en Andalucía (España) planteó la necesidad de localizar una especie que, como Allium rouyi, ha sido considerada como extinta por diversos autores (Gómez Campo et al. 1987; Moreno Saiz y Sainz Ollero, 1992) y que como tal aparece en diversos listados nacionales e internacionales.

Esta especie fue descrita por Gautier (Rouy, 1898) con material recolectado en la sierra de Estepona (Málaga, España) en el año 1896. Desde entonces no había vuelto a ser recolectada a pesar de las numerosas campañas realizadas. El único material existente hasta el momento es el pliego del tipo (Stearn, 1978) conservado en el herbario de Rouy en Lyon (LY). El interés se centró, por tanto, en intentar encontrar alguna población de esta especie y, en caso afirmativo, elaborar un plan para su recuperación y conservación.

\section{MATERIAL Y MÉTODOS}

En base a la fecha de recolección del material tipo y conociendo la ecología y fenología de otras especies de Allium localizadas en el sector Bermejense, se programó una búsqueda sistemática durante los meses de Mayo y Junio de 1992. Como resultado de las mismas fue localizada una población de Allium rouyi, de areal muy reducido, formada por medio centenar de individuos, de los que fueron recolectados 3 ejemplares con bulbos. En Julio de 1992 se recolectaron semillas con el fin de iniciar estudios sobre germinación. La mayoría de los ejemplares observados presentaban bulbillos pedunculados que se situaban por encima del bulbo principal. El número de flores y la altura de las plantas fueron medidas en todos los individuos de la población.

Para el estudio polínico se ha utilizado material fresco, recién recolectado,

Trabajo realizado en el marco del convenio, sobre especies vegetales amenazadas, firmado entre la Agencia de Medio Ambiente de la Junta de Andalucía y la Universidad de Málaga. 
incluido en ácido acético glacial, siendo posteriormente las muestras acetolizadas según el método de Erdtman (1960) posteriormente modificado por Reitsma (1969). El montaje para la observación de los granos de polen con el microscopio óptico se realizó utilizando como medio glicerogelatina incolora y, para realizar las microfotografías electrónicas, las muestras fueron recubiertas con oro vaporizado en atmosfera de alto vacío. Igualmente se utilizó el MEB para el estudio de la superficie de las semillas.

\section{RESULTADOS} especie:

Con el material recolectado se ha realizado una nueva descripción de la Allium rouyi Gaut. in Rouy, Ill. Pl. Eur. Rar. 10: 81 (1898)

Bulbo de 1,5-2 x 0,8-1,2 cm, ovoideo a elipsoideo; túnica externa membranosa, haciéndose fibrosa en la parte superior; fuertemente oloroso. Bulbillos numerosos de (5-) 6-8 x (3-) 4-5 (-5.5) mm, elipsoideos, con estípites, generalmente sobrepasando el bulbo. Tallo de (15-) 18-30 (-50) cm, de sección circular. Hojas (2-) 3-5, de hasta 17 x 0,05-0,10 cm, más cortas que el tallo, semicilíndricas a filiformes, envainando 1/4-1/3 del tallo; glabras o con pelos reflejos, generalmente caducos, de hasta 1,5-2 $\mathrm{mm}$, en vainas y márgenes. Espata con 2 piezas desiguales, de 0,6 - $1 \mathrm{~cm}$, más cortas que la inflorescencia, agudas y persistentes. Inflorescencia de 3-4,5 x 2-3 cm, laxa, con (2-) 5-15 (-22) flores, con bractéolas entre los pedicelos florales; pedicelos de 0,5$3 \mathrm{~cm}$. Perigonio campanulado. Tépalos amarillento-verdoso, con nervio medio verdoso; pasando a pardo-amarillento con nervio verdoso al secarse; los externos de (3,5-) 56 × 2-2,5 mm, ovados a oblongos, obtusos; los internos de (3-) 5-5,5-(6) x 1,5-2 (-2,5) $\mathrm{mm}$, oblongos, obtusos; ambos pareciendo agudos al secarse. Estambres incluidos o ligeramente exertos; filamentos amarillos, simples, de (4-) 4,5-5,5 (-6) mm; anteras amarillas; polen amarillo. Ovario globoso; estilo de (1,5-) 2 - $3(-3,5) \mathrm{mm}$; estigma de linear a capitado. Capsula de 3,5 - $4(-4,5) \mathrm{mm}$, globosa. Semillas de 3-3,6 x 1,4 - 2,1 $\mathrm{mm}$.

Distribución: endemismo del sector Bermejense (Nieto Caldera et al. 1991); Sierra Bermeja (Estepona, Málaga).

Ecología: edafoendemismo serpentinícola localizado en el areal de la serie del Pino pinastri-Querceto cocciferae (Cabezudo et al. 1989).

Morfología polínica: polen monosulcado, heteropolar, bisimétrico. Elíptico en vista polar y corte óptico ecuatorial; plano-convexo en vista ecuatorial y corte óptico meridiano; transverso, siendo $\mathrm{P} / \mathrm{E}=0,50-0,66(\overline{\mathrm{x}}=0,59 \pm 0,04)$. Tamaño mediano, con valores de $\mathrm{P}=18-22(\overline{\mathrm{x}}=19,32 \pm 0,97) \mu \mathrm{m}$, de $\mathrm{E}_{1}=29-36(\overline{\mathrm{x}}=32,66 \pm 1,56) \mu \mathrm{m}$ y de $\mathrm{E}=17-23(\overline{\mathrm{x}}=20,44 \pm 1,15) \mu \mathrm{m}$. Abertura simple, de tipo sulco, que recorre todo el polo distal. Exina de aproximadamente $1,5 \mu \mathrm{m}$ de grosor en el polo proximal, con la sexina ligeramente más gruesa que la nexina. Téctum perforado. Infratéctun columelado. Superticie rugulado-perforada (Lám. 1, figs. 1, 2 y 3).

Morfología de las semillas: semillas de sección longitudinal hemielíptica, ligeramente cóncavo-convexas o aplanadas, con el dorso redondeado y la base plana, presentando una escotadura semilunar en uno de los extremos en cuyo interior se sitúa el hilo, que es blanquecino. Testa de color negro formada por células coliculadas poligonales de borde ligeramente ondulado, con un tubérculo central de $10-20 \mu \mathrm{m}$ de diámetro rodeado de otros más pequeños. La región intercelular aparece más o menos estriada (Lám. 1, figs. 4 y 5).

Material estudiado: Málaga, Estepona, Sierra Bermeja, 9.VI.1992, Cabezudo, 


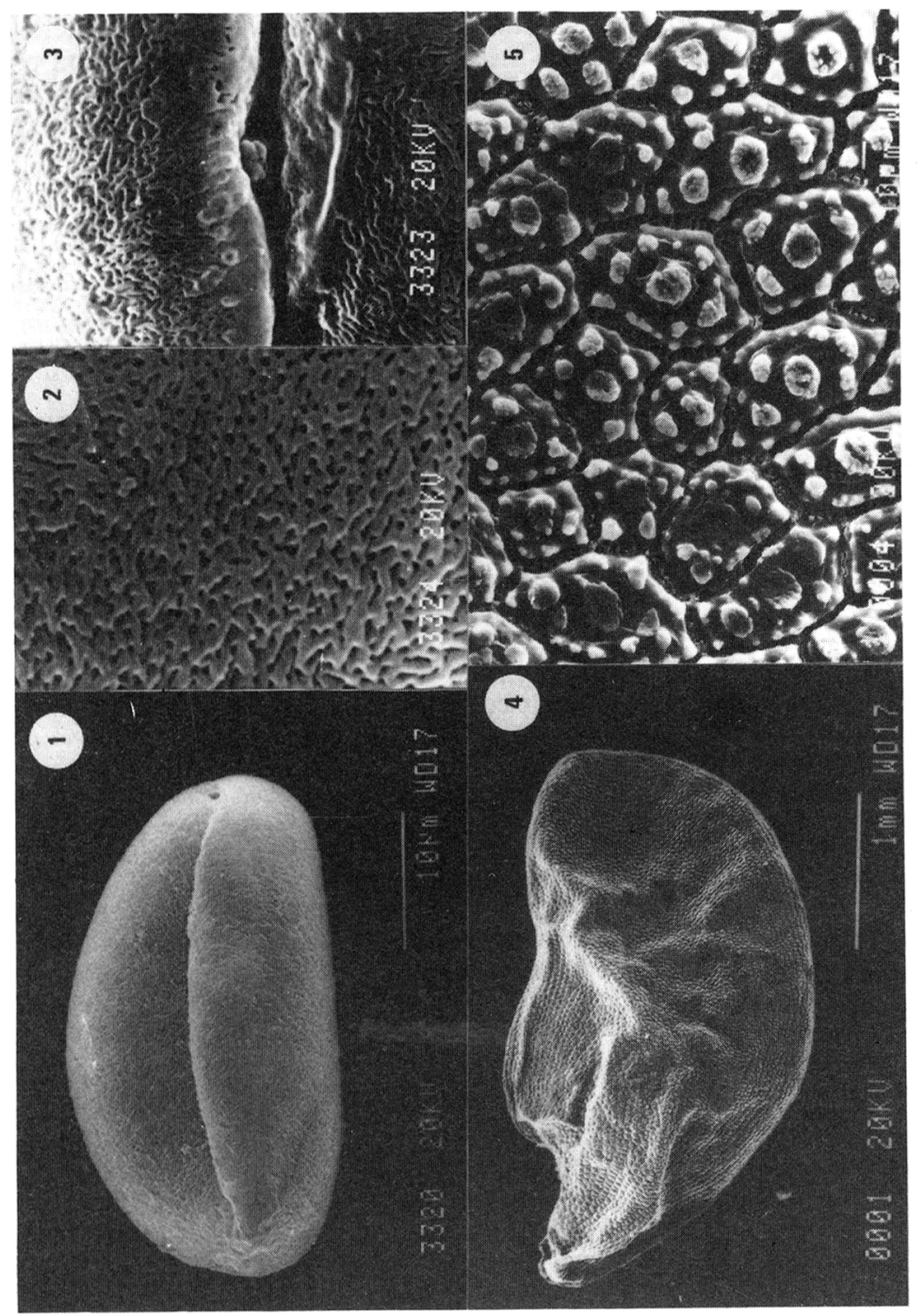


Pérez-Latorre, Navas y Recio; nº 208/92 (MGC 35.000); idem, 11.VI.1992, Cabezudo, Navas y Nieto Caldera; nº 213/92 (MGC 35.001). Semillas recolectadas el 7 de julio de 1992.

\section{DISCUSIÓN}

Esta especie se incluye en la sect. Scorodon por una serie de caracteres como su túnica membranosa-fibrosa, hojas semicilíndricas a filiformes, espata con dos piezas cortas y desiguales, perigonio campanulado y estambres simples. No obstante, resulta singular la presencia de bulbillos estipitados, ya que no es frecuente en esta sección, aunque sí en otras como la sect. Allium o Molium (Pastor, 1983).

Los resultados del análisis polínico coinciden, en morfología y tamaño de ejes, con los de otras especies estudiadas de la sección, como A. moschatum o A. grosii (Pastor, 1982).

Las observaciones de las semillas permiten situar a $A$. rouyi, por sus células de la testa coliculadas y con tubérculos de distinto tamaño, juno a A. chrysonemum. Otras especies de la sección con datos conocidos de la semilla, como A. moschatum (Pastor, 1981) tienen las células de la testa planas y con la superficie granulada.

Por los caracteres morfológicos y de las semillas, fundamentalmente, en las especies de la sect. Scorodon pueden diferenciarse dos grupos: por un lado $A$. moschatum, A. hirtovagiratum y A. grosii; y por otro A. chrysonemum y A. rouyi, los dos táxones de la sección que se distribuyen más al sur de la Península Ibérica y que constituyen notables endemismos de Andalucía.

\section{BIBLIOGRAFÍA}

CABEZUDO, B., J. M. NIETO CALDERA y A. V. PÉREZ-LATORRE -1989- Contribución al estudio de la vegetación edafófilo serpentinícola del sector rondeño(Málaga, España). Acta Bot. Malacitana, 14: 291-294.

ERDTMAN, G. -1960- The acetolysis method. Svensk. Bot. Tidskr. 54: 561-564.

GÓMEZ CAMPO, C. in GÓMEZ CAMPO, C et al. -1987-Libro Rojo de las Especies Vegetales Amenazadas de España Peninsular e Islas Baleares. I. C. O. N. A.

MORENO SAIZ, J. C. y H. SAINZ OLLERO -1992-Atlas corológico de las monocotiledoneas endémicas de la Península Ibérica e Islas Baleares. Madrid.

NIETO CALDERA, J. M., A. V. PÉREZ-LATORRE y B. CABEZUDO -1991- Biogeografía y series de vegetación de la provincia de Málaga (España). Acta Bot. Malacitana, 16: 417-436.

PASTOR, J. -1981-Contribución al estudio de las semillas de las especies de Allium de la Península Ibérica e Islas Baleares. Lagascalia, 10: 207-216.

PASTOR, J. - 1982- Contribución al estudio palinológico del género Allium en la Península Ibérica. Bot. Macaronesica, 8-9: 189-214.

PASTOR, J. y B. VALDÉS -1983-Revisión del género Allium en la Península Ibérica e Islas Baleares. Anales Univ. Hispalensis. Sevilla.

REITSMA, T. -1969- Size modofication of recent pollen grains under different treatments. Rev. Palaeobot. Palynol. 9: 175-202.

ROUY, G. C. C. -1898- Illustrationes plantarum Europae rariorum. 10: 81.

STEARN, W. T. -1978- European species of Allium and allied genera of Alliaceae: a synonymi enumeratio. Ann. Musei Goulandris, 4: 83-198.

(Aceptado para su publicación el 5 de Agosto de 1992)

Dirección de los autores: B. Cabezudo, M. Mar Trigo y J.M. Nieto Caldera, Departamento de Biología Vegetal. Facultad de Ciencias. Universidad de Málaga. J. Pastor, Departamento de Botánica y Ecología. Facultad de Ciencias. Universidad de Sevilla. 